\title{
The Revelations of Acute Kidney Injury in Cases of Acute Febrile Illness - A Hospital Based Observational Study from North Eastern India
}

\author{
Kallol Bhattacharjee ${ }^{1 \star}$, Sanjeeb Roy ${ }^{2}$, P. Balamurali ${ }^{2}$, Nabaruna Paul ${ }^{2}$
}

${ }^{1}$ Professor, Department of Medicine, Silchar Medical College and Hospital

${ }^{2} P G T$, Department of Medicine, Silchar Medical College and Hospital

\author{
Corresponding author \\ Kallol Bhattacharjee, Professor, Department of Medicine, Silchar Medical \\ College and Hospital, Silchar, Assam, India
}

Submitted: 27 Jan 2020; Accepted: 30 Jan 2020; Published: 05 Feb 2020

\begin{abstract}
Background: Clinicians across the globe refer to the published KDIGO definition of Acute Kidney Injury (AKI) as one of the following:

- An increase in serum creatinine by $\geq 0.3 \mathrm{mg} / \mathrm{dl}(\geq 26.5 \mu \mathrm{mol} / \mathrm{l})$ within $48 \mathrm{hrs}$

- An increase in serum creatinine to $\geq 1.5$ times baseline within the previous 7 days

- Urine volume $<0.5 \mathrm{ml} / \mathrm{kg} / \mathrm{h}$ for $6 \mathrm{hrs}$

Acute febrile illnesses are a common cause of AKI in hospitalized patients. The present study was undertaken to evaluate the incidence of AKI in patients presenting with acute febrile illness and also study the different etiological factors responsible for acute febrile illness.
\end{abstract}

Materials and Methods: The study included 200 patients of acute febrile illness admitted in Silchar Medical College And Hospital in the Department of Medicine over a period of 24 months. The data regarding the various causes such as the etiology of fever, kidney function tests and other parameters of the cases were obtained and analyzed using simple statistical methods.

Results and Observations: A total of 52 patients (26\%) with acute febrile illness due to etiologies like Leptospirosis, Falciparum Malaria, Enteric fever, Dengue, Scrub Typhus, and mixed Malaria, etc developed AKI out of the 200 admitted cases presenting with acute febrile illness.

Conclusion: The incidence of AKI is common in hospitalized patients of acute febrile illness and a thorough evaluation and detailed clinicobiochemical monitoring of the patients are necessary as it has varied etiology and often lead to an unfavorable or even unexpected outcome.

Keywords: Acute Kidney Injury, AKI, Serum Creatinine, Febrile, Patients, Leptospirosis

\section{Introduction}

Acute kidney injury (AKI) has dominated the attention of nephrologists for decades, with research aimed at reducing the morbidity and mortality related to the entity. AKI has been a subject of interest for the physicians as well as nephrologists in the tropical countries owing to a very high burden of disease related to infectious causes. Care for this category of patients involves an aggressive approach by a combination of the management of infectious aetiology and the renal care. AKI caused by infective as well as non-infective causes are a serious complication and encompasses a serious threat to life of an individual suffering from it. And above all, it is an important cause of death in the patients diagnosed with acute febrile illness.

Nephrologists all over the globe universally accept and practice the published KDIGO definition of Acute Kidney Injury as any one stated below [1].

- An elevation of serum creatinine by $\geq 0.3 \mathrm{mg} / \mathrm{dl}(\geq 26.5$ umol/l) within $48 \mathrm{hrs}$

- An elevation of serum creatinine to $\geq 1.5$ times baseline within the previous 7 days

- Urine output $<0.5 \mathrm{ml} / \mathrm{kg} / \mathrm{h}$ for $6 \mathrm{hrs}$

Acute febrile illnesses are quite common occurrences in the community and most of the cases are seen by local health care practitioners and treated empirically with drugs such as anti-malarial and antibiotics in most of the cases. Subsequently they are referred to or find their way to tertiary referral hospitals owing to an inadequate response to these medications. Acutely presenting undifferentiated febrile illness comprises a major chunk of the patients presenting to the physicians in a tertiary care hospital. Acute febrile illness is one of the commonest etiologies of AKI in hospitalized patients. 
Recent studies show AKI occurring in patients diagnosed with scrub typhus, typhoid, leptospirosis, malaria, dengue fever and acute undifferentiated febrile illness.

\section{Aims and Objectives}

This study was done to evaluate the incidence of AKI in patients presenting with acute febrile illnesses and also study the different etiological factors responsible for febrile illness of acute origin in Barak Valley region of Assam in the remote north eastern corner of India.

Materials and Methods

The research work was a prospective observational study that included 200 patients of acute febrile illness admitted in Silchar Medical College and Hospital in the Department of Medicine over a period of 24 months from 1st July 2017 to 30th June 2019.

\section{Inclusion Criteria}

- Inpatient

- Age $>18$ yrs

- Duration of fever 5-21 days

- Patients agreeing to undertake written and informed consent

Exclusion Criteria

- Localized infection

- HIV infection

- Autoimmune disease

- Presence of other co morbid conditions

- Previous history of kidney diseases or pre existing kidney diseases

- Malignancy

- Patients refusing to give written and informed consent.

- Patients on nephrotoxic agents

All patients had undergone a detailed clinical evaluation and the necessary investigations including a complete blood count, liver function tests, kidney function tests, chest skiagram, 3 peripheral blood smears for malarial parasite, a detailed urinalysis, 2 blood culture, and the recommended standard set of febrile serological investigations that included leptospiral IgM ELISA, scrub typhus ELISA, dengue NS1 ANTIGEN, IgM \& IgG ELISA. In cases whose preliminary tests were insufficient to arrive at the final diagnosis, further tests including convalescent serological testing were performed. All patients were observed till the entire period of hospitalisation. Serum creatinine was measured using the automated chemistry analyser VITROS 5600.

\section{Results and Observations}

A total of 52 patients (26\%) with acute febrile illness due to Enteric fever, Falciparum Malaria, Dengue, Scrub Typhus, Leptospirosis and Mixed Malaria developed AKI out of the 200 admitted cases presenting with acute febrile illness. Out of the 200 cases the different etiologies of fever are as follows.
Table 1: Etiologies of Acute Febrile Illness

\begin{tabular}{|l|c|}
\hline Etiology & No. of cases \\
\hline Vivax Malaria & 38 \\
\hline Falciparum Malaria & 24 \\
\hline Mixed Malaria & 6 \\
\hline Dengue fever & 8 \\
\hline Enteric fever & 38 \\
\hline Leptospirosis & 42 \\
\hline Scrub typhus & 18 \\
\hline Undifferentiated fever & 26 \\
\hline
\end{tabular}

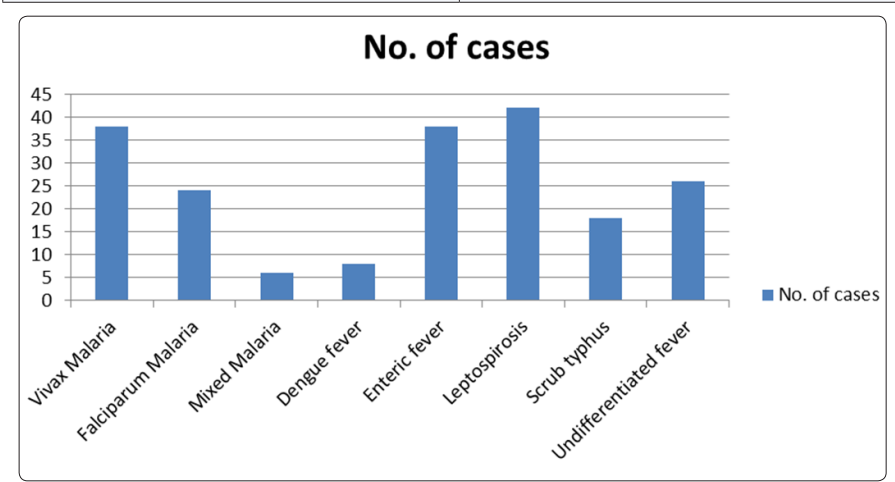

Figure 1: Distribution of different etiologies of Acute Febrile Illness

Table 2: Sex Distribution of the cases

\begin{tabular}{|l|c|}
\hline MALES & FEMALES \\
\hline 132 & 68 \\
\hline
\end{tabular}

\section{Sex Distribution}

- Males Eemales

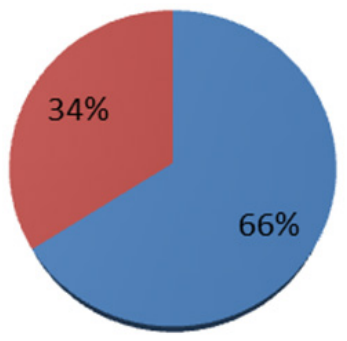

Figure 2: Sex Distribution of the cases 
Table 3: Incidence of AKI among the study population according to etiology

\begin{tabular}{|l|c|c|}
\hline Disease & $\begin{array}{c}\text { No. of cases } \\
\text { developing AKI }\end{array}$ & $\begin{array}{c}\text { Percentage of cases } \\
\text { developing AKI }\end{array}$ \\
\hline Leptospirosis & 25 & $59.5 \%$ \\
\hline Scrub typhus & 4 & $22.2 \%$ \\
\hline Enteric fever & 4 & $10.5 \%$ \\
\hline Dengue fever & 1 & $12.5 \%$ \\
\hline Vivax Malaria & 4 & $10.5 \%$ \\
\hline Falciparum Malaria & 9 & $37.5 \%$ \\
\hline Mixed Malaria & 1 & $16.6 \%$ \\
\hline Undifferentiated fever & 4 & $15.3 \%$ \\
\hline
\end{tabular}

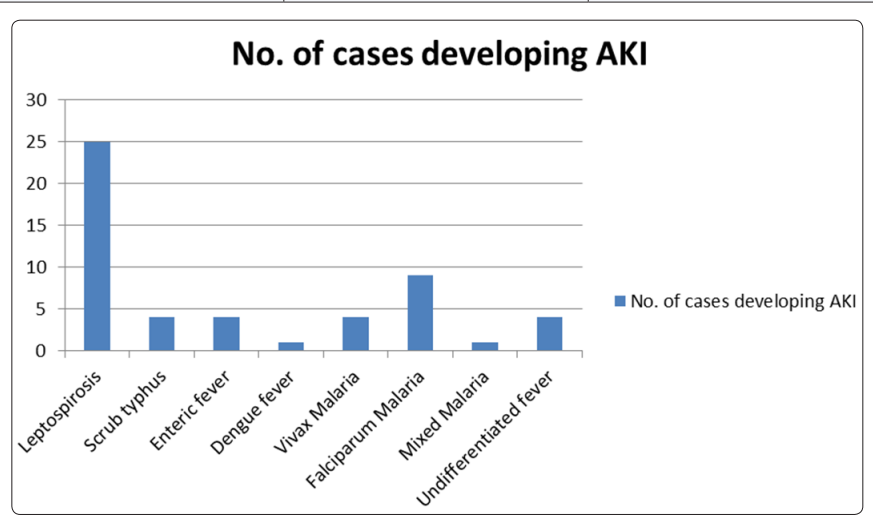

Figure 3: Number of cases developing AKI according to etiology

Table 4: Sex distribution of the cases developing AKI

\begin{tabular}{|l|c|}
\hline MALES & FEMALES \\
\hline 32 & 20 \\
\hline
\end{tabular}

\section{Sex Distribution of AKI cases}

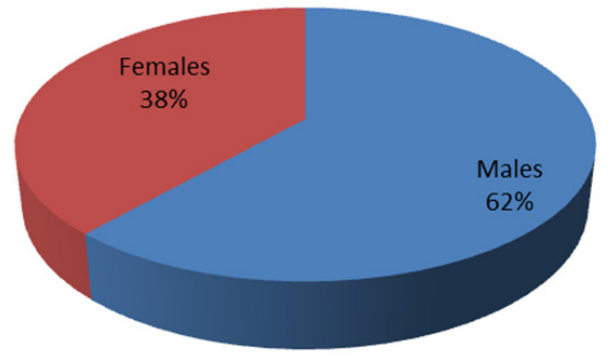

Figure 4: Sex distributions of AKI cases

Total number of patients that required renal replacement therapy (RRT) was 5 and the in hospital mortality among the cases of AKI was 2 . Out of the 2 cases of in hospital mortality 1 case was of mixed malaria and the other one was a case of Dengue fever that subsequently developed Dengue Shock Syndrome.
Table 5: Requirement of RRT according to the etiology of Acute Kidney Injury

\begin{tabular}{|l|c|}
\hline Disease & No. of cases requiring Hemodialysis \\
\hline Leptospirosis & 1 \\
\hline Scrub typhus & 0 \\
\hline Enteric fever & 1 \\
\hline Dengue fever & 1 \\
\hline Vivax Malaria & 0 \\
\hline Falciparum Malaria & 1 \\
\hline Mixed Malaria & 1 \\
\hline Undifferentiated fever & 0 \\
\hline
\end{tabular}

The commonest etiology of Acute febrile illness was Leptospirosis (21\%) followed by Vivax Malaria (19\%). The commonest etiology of AKI observed in this study was Leptospirosis. The highest proportion of AKI occurred in Leptospirosis at 59.5\%.

\section{Discussion}

The incidence of AKI is common in hospitalized patients of acute febrile illness. The present series showed the incidence of AKI as $26 \%$ compared to $41.1 \%$ in the study done by G.Basu et al, $13.21 \%$ in K.Mehta et al and 54\% in Jayalal Jayapalan Nair et al [2-4]. In our study male preponderance $(62 \%)$ was observed in AKI as regards incidence in patients presenting with acute febrile illness which is similar to the results obtained by Jayalal Jayapalan Nair et al (more than 75\% ), Dr Arjun.V. A et al (62\%) and G.Basu et al (64.2\%) [5]. The commonest cause of AKI in our study was Leptospirosis which is in contrast to G.Basu et al. that showed Falciparum malaria as the commonest etiology. However our study revealed similar results as Jayalal Jayapalan Nair et al. and Rasis Wan Ahmad Kamil et al [6]. That revealed Leptospirosis as the commonest etiology of AKI. The commonest etiology for Malaria associated AKI was P. falciparum which is similar to the results obtained by Kallol Bhattacharjee et al [7]. In the series by Hwang $\mathrm{K}$ et al, the feature of AKI in scrub typhus was $35.9 \%$ whereas in our study it was slightly lesser at $22.2 \%$ [8]. The requirement of RRT was in $9.6 \%$ cases of AKI which was marginally higher in comparison to G.Basu et al at $7.9 \%$. The in hospital mortality in the patients developing AKI was 3.8\% similar to Jayalal Jayapalan Nair et al at 3\%.

\section{Conclusion}

AKI in acute febrile illness is common often requiring multispeciality care with dialysis and hemodynamic as well as ventilator support preferably in an ICU setup. Mortality is high with shifting trends in management from wards to ICUs. Emphasis on prevention and management at the earliest point of contact, particularly the community level by educating registered medical practitioners on acute febrile illness pattern recognition, renal function assessment and early hydration, appropriate antibiotic use, avoidance of nephrotoxic drugs, nutrition and early referral to nephrologists for patients with suspicion of AKI will reduce the burden of disease in the long run. Malaria, leptospirosis, enteric fever, scrub typhus, dengue and undifferentiated fever were the common causes of fever in the present study. The incidence of AKI among the patients in the present study was $26 \%$. AKI is undoubtedly a significant risk factor for mortality. An aggressive approach to arrive at a diagnosis and the early initiation of therapy should be the focus of the clinicians to obtain a clinical outcome which is one of the best in the present days across the globe. 
Limitations of the Study

The study was a single centered observational study of short duration. A multi centric study covering a wider geographical area and representing diverse ethnic groups of the society, done for a longer duration would have been ideal for this study.

\section{References}

1. KDIGO GUIDELINES 2012

2. Basu G, Chrispal A, Boorugu H, Gopinath KG, Chandy S, et al. (2011) Acute kidney injury in tropical acute febrile illness in a tertiary care centre-RIFLE criteria validation. Nephrology Dialysis Transplantation 26: 524-531.

3. Mehta K, Pajai A, Bhurke S, Shirkande A, Bhadade R, et al. (2018) Acute kidney injury of infectious etiology in monsoon season: A prospective study using acute kidney injury network criteria. Indian J Nephrol 28: 143-152.

4. Nair, Jayalal Jayapalan, Ajay Bhat, Mangalore Venkatraya
Prabhu (2016) "A Clinical Study of Acute Kidney Injury in Tropical Acute Febrile Illness". Journal of Clinical and Diagnostic Research 10: OC01-OC05.

5. Arjun. V A, Sachin Bongale, K Siddappa (2018) Clinical Profile of Acute Kidney Injury in Acute Febrile Illness with Thrombocytopenia. Clinical Profile of Acute Kidney Injury in Acute Febrile Illness with Thrombocytopenia 6: 489-494.

6. http://www.kireports.org/article/S2468-0249(17)30225-5/pdf

7. Kallol Bhattacharjee, Abhishrut Jog, Chandra Prakash Thakur, Dwijen Das (2018). The Revelations of Malaria Associated Kidney Disease - A Menace in the Tropics. JOJ Urology \& Nephrology 5: 555659.

8. Hwang K, Ha Nee Jang, Tae Won Lee, Hyun Seop Cho, Eunjin Bae et al. (2017) Incidence, risk factors and clinical outcomes of acute kidney injury associated with scrub typhus: a retrospective study of 510 consecutive patients in South Korea (2001-2013). BMJ Open 7: 1-7.
Copyright: (C2020 Kallol Bhattacharjee, et al. This is an open-access article distributed under the terms of the Creative Commons Attribution License, which permits unrestricted use, distribution, and reproduction in any medium, provided the original author and source are credited. 